\title{
Determinants of Low Antenatal Care Services Utilization during the First Trimester of Pregnancy in Southern Benin Rural Setting
}

\author{
Ouendo Edgard-Marius*, Sossa Jerome Charles, Saizonou Jacques, Guedegbe Capo-Chichi Justine, \\ Mongbo Ade Virginie, Mayaki Alzouma Ibrahim, Ouedraogo T. Laurent
}

Department of Policy and Health Systems, Regional Institute of Public Health, Benin

Copyright (C) 2015 by authors, all rights reserved. Authors agree that this article remains permanently open access under the terms of the Creative Commons Attribution License 4.0 International License

\begin{abstract}
Antenatal care services for pregnant women improve maternal and child health outcomes. It is recognized that antenatal care services are underutilized in developing countries. The objective of the study was to identify the determinants of low utilization of antenatal care services during the first trimester of pregnancy in southern Benin rural setting. The cross-sectional and analytical study included 301 pregnant women, 29 husbands and 21 health workers in Athiémé municipality (southern Benin rural setting) and was conducted from 14 April to 14 July 2014. Data on sociodemographic and antenatal services characteristics were collected using questionnaire, semi-structured interviews and focus groups. Determinants of low utilization of antenatal care services were identified using logistic regression model. Among 301 pregnant women, only $24.6 \%$ utilized antenatal care services during the first trimester of the pregnancy. Five independent determinants of low utilization of antenatal care services were identified: low education level of pregnant women, $\mathrm{OR}=3.3[95 \% \mathrm{CI}=(1.534: 7.071)]$; wrong knowledge of the required period for the first attendance to antenatal care service during pregnancy, $\mathrm{OR}=5.131 \quad[95 \% \mathrm{CI}=$ (1.972:13.353)]; adequate knowledge of the benefits of the antenatal care service, $\mathrm{OR}=4.031[95 \% \mathrm{CI}=(1.401: 11.594)]$; participation in the behavior change communication sessions, $\mathrm{OR}=0.059 \quad[95 \% \mathrm{CI}=\quad(0.028: 0.125)] ; \quad$ adequate patient-welcome in antenatal care services, $\mathrm{OR}=0.162$ $[95 \% \mathrm{CI}=(0.051: 0.513)]$. Sociocultural reasons were also mentioned. Pregnant women underutilize free antenatal care services in Athiémé municipality. Effective interventions taking into account the identified determinants may contribute to improve the utilization of antenatal services in southern Benin rural setting.
\end{abstract}

Keywords Determinants, Service Utilization, Antenatal Care, Pregnancy First Trimester, Benin

\section{Introduction}

Antenatal care (ANC) is one of the four pillars of the Initiative for Safe Motherhood [1]. The main objectives of ANC are: prevention and treatment of obstetric complications, preparation for emergencies, family planning, meeting nutritional, social, emotional needs for pregnant woman, including care and nutrition of the newborn [2].

Women attending ANC visits receive sufficient evidence-based clinical interventions, such as tetanus toxoid immunization, deworming, iron and folic acid supplements, counseling on maternal health, emergency preparedness, management of sexually transmitted infections, administration of antiretroviral therapy in HIV-positive women, supply of essential information about improved hygienic practices, and the risks associated with pregnancy and childbirth [3].

The World Health Organization (WHO) recommends that pregnant women in developing countries initiate early prenatal care before the end of the $4^{\text {th }}$ month of pregnancy [4]. ANC for the first trimester is fundamental and decisive to identify and evaluate the risk factors usually present before pregnancy [4].

An analysis of Demographic and Health Surveys in 45 developing countries showed that women in sub-Saharan Africa utilized prenatal care much later than women in other regions [5]. In Benin, less than one out of two women (48\%) completed her first prenatal visit before the fourth month of pregnancy [6]. In $28 \%$ of cases, pregnant women waited 4-5 months before attending ANC provide by skilled health workers [7].

Worldwide, several factors including the place of residence of women, ethnicity, education, occupation, economic status, and distance to ANC services influence the timing of the first ANC attendance [3]. In developing countries, the three delay model especially affecting antenatal attendance and reference includes the mothers' failure to have health 
seeking behavior, failure to access services and failure to obtain appropriate and timely care [8]. Marital status, ANC availability, household income, media exposure, and family social support [2] were reported to contribute to the three delays in addition to above factors [8]. ANC commenced early provides preventive strategies, opportunities for assessment of pregnant mothers, management of obstetric complications or appropriate timely referrals aimed at reduction of maternal and perinatal morbidity and mortality.

The quality of antenatal care, beliefs and perceptions about pregnancy may have an influence on the utilization of ANC, explaining early or late attendance [2]. There is currently little data available at the national level, on obstacles and barriers to the utilization of ANC services by pregnant women during the first trimester of pregnancy. This study addresses this problem with the aim to identify the determinants of low utilization of ANC services in the first trimester of pregnancy in pregnant women in a southern Benin rural setting in 2014.

\section{Materials and Methods}

\subsection{Conceptual Framework of the Utilization of Antenatal Care Services in the First Trimester of Pregnancy in Southern Benin Rural Setting}

The proposed conceptual framework (Figure 1) explains the relationship between the low utilization of ANC services in the first trimester of pregnancy and its potential explanatory factors according to the literature data. The demographic and socio-cultural factors related to pregnancy, socio-cultural factors linked to pregnancy, factors related to accessibility to health services and their characteristics explain the low utilization of ANC in the first trimester of pregnancy and are also inter-related.

\subsection{Setting}

The study was conducted in the municipality of Athiémé, located in the Department of Mono in the southern Benin. The municipality of Athiémé also called commune of Athiémé is a decentralized territorial division that corresponds to a group of communities. It covers an area of $238 \mathrm{Km}^{2}$ with estimated population at 56247 habitants [7]. Customary marriage remains popular in the locality. Based on a traditional model, women in Athiémé municipality play a secondary role despite their involvement in the economic activities of the household and the city [9]. Five complete health centers (nurse clinic and maternity clinic together), three isolated nurse clinics (nurse clinic alone), and four isolated centers for maternal health (maternity clinic alone) are available. The isolated nurse clinics don't provide ANC.
Besides ANC, complete health centers and maternity clinic alone provide immunization, contraception, deliveries and postnatal care. The working days and hours are $24 \mathrm{~h}$ daily. There are no other outreach services providing antenatal care in the community. Mothers who attend ANC have a mother child booklet that they take home. The elements of antenatal care given include prenatal visit, tetanus toxoid immunization, deworming, iron and folic acid supplements, counseling on maternal health, emergency and breastfeeding preparedness, management of sexually transmitted infections, administration of antiretroviral therapy in HIV-positive, supply of essential information about the risks associated with pregnancy, childbirth and improved hygienic practices and referral system from clinic if required. Usually men do not accompany women to motherhood clinic. For all of the commune of Athiémé, 29 health workers involved in the ANC including 12 skilled employees (nurses and midwives) and 17 non-qualified caregivers.

\subsection{Study Design}

This is a cross-sectional descriptive and analytic study using mixed methods to examine the determinants of low utilization of of ANC services by pregnant women during the $1^{\text {st }}$ trimester. The study was conducted during the period 14 April to the 28 July 2014

\subsection{Study Population}

The primary target population was pregnant women attending their ANC visit regardless of the length of the pregnancy. Health care providers including midwives involved in isolated maternal health centers and husbands of pregnant women were secondary targets populations.

\subsection{Sample Size and Selection of Target Populations}

To determine minimum required sample size, we use "knowledge of required period of the first ANC visit for pregnant women" as the principal independent variable. Then, the sample size required to detect Odd ratio of 02 with statistical power of $0.80, \alpha=0.05$ using logistic regression was 300 [10].

All pregnant women regardless of rank and length of the pregnancy as they come for ANC visit were selected and interviewed after their consent.

Care providers including midwives who were present the days of interviews were included in the study.

A convenience sample of husbands was recruited in community from men whose wives have given birth once.

Overall, a total of 301 pregnant women, 19 health care providers, two matrons and 29 husbands participated in in the study. 


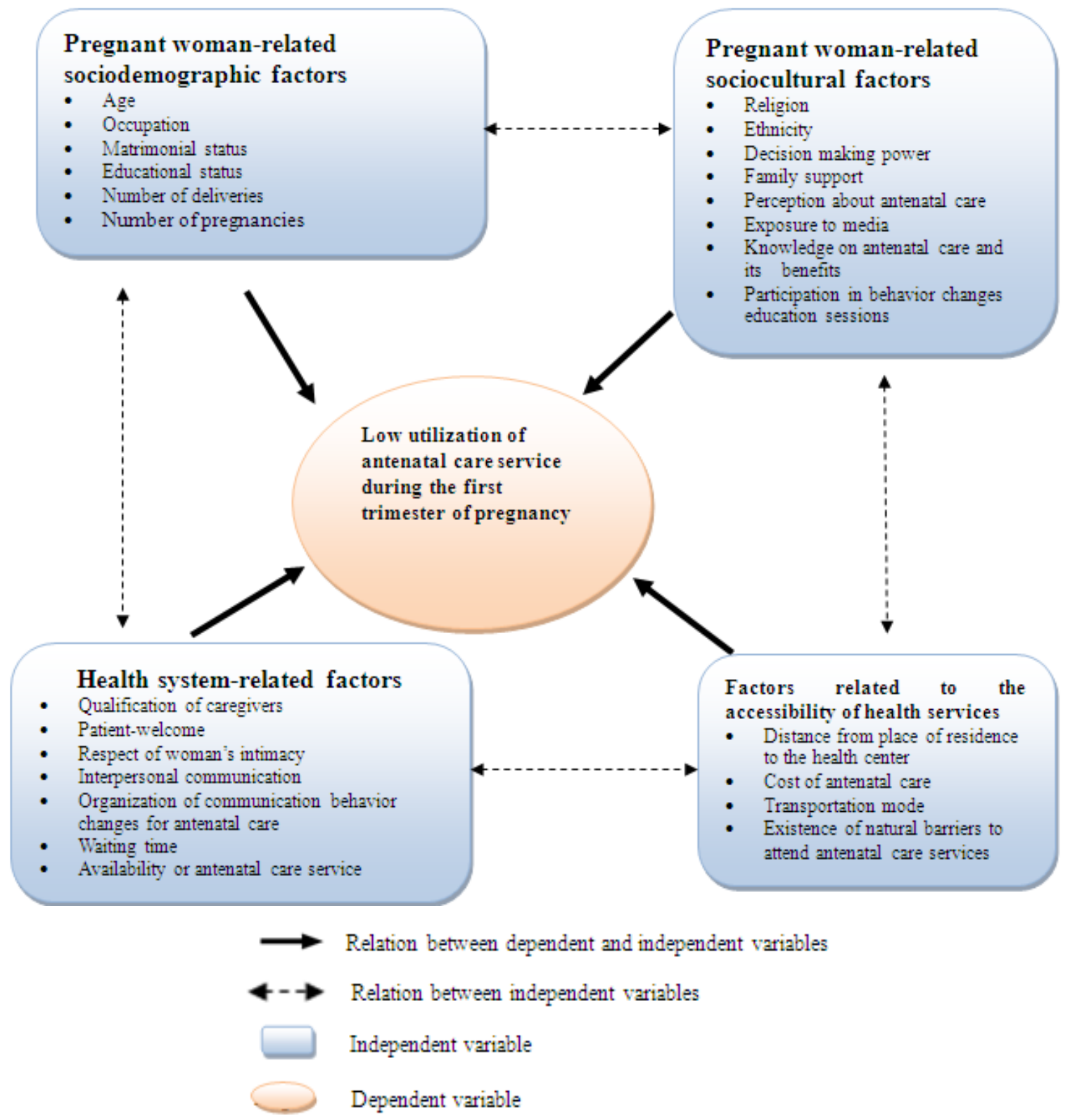

Figure 1. Conceptual framework of low utilization of antenatal care service during the first trimester of pregnancy in southern Benin in 2014.

\subsection{Study Variables}

The dependent variable was the low utilization of antenatal care services during the $1^{\text {st }}$ trimester of pregnancy.

The independent variables were: demographic variables, sociocultural characteristics related to the pregnant woman; factors related to accessibility and health services as shown in the conceptual framework figure1. Behavioral change communication sessions refer to out of health centers meeting that aim at advising women by mid-wife on antenatal and postnatal care utilization.

\subsection{Data Collection Procedures}

For data collection, four techniques were used namely: questionnaire survey, semi-structured interviews, focus groups and direct observation. Pregnant women were surveyed using questionnaires relative to sociodemographic characteristics and first ANC visit. We use individual interviews to collect data in health care providers including matrons. Data were collected in husbands during three different focus groups. Data were collected by trained enumerators. Data collection tools were pre-tested for required adaptations. Items on data collection tools explored factors showed on conceptual frame. Compositions of items were continuous for quantitative data and were categorical according to qualitative data. Focus groups aimed at discussing on determinants of low ANC utilization. Focus group duration was one hour and took place in consented location (meeting room). Regular supervision helped to ensure that the collection procedures were properly followed.

\subsection{Data Analysis}


Stata software, version 11 and Excel were used for data analysis. Quantitative variables were described with their averages and standard deviations. Categorical variables were described by calculating frequencies.

The statistical test of Chi-square of Pearson was used to compare proportions of pregnant women with low utilization of ANC during the first trimester of pregnancy according to independent variables modalities. Student's test was used to compare means. Multivariate logistic regression model was used to identify determinants of the low utilization of ANC during first trimester of pregnancy. Odds Ratios and confidence intervals were used to assess the relation between determinants and ANC utilization. Level of signification was set at $\mathrm{p}<0.05$.

Data collected by focus group are used to point out the factors that lead husbands not to encourage women to go to prenatal consultation in the first trimester of the pregnancy. Among these factors, those that did not already emerged by qualitative analyzes were taken into account.

\subsection{Ethical Considerations}

The objectives of the study were explained to participants. Voluntary informed consent was obtained from each participating woman before starting the interview. This consent claims that participants were not at risk by refusing to participate in the survey or stopping their collaboration during the study and would not have their maternity care impacted in any way prior to commencing the interview. Those who did not consent were provided with standard of care. The confidentiality and anonymity of the information collected were respected. After the interview, benefits of completing four ANC or more were explained to pregnant women. We have obtained the approval of the responsible officers from health districts and local authorities of the Athiémé municipality before the investigation started.

\section{Results}

Among pregnant women approached, eight of 309 refused to participate in the study on market days because they had no time for interview and wanted to go to sell at the market. The success rate was $97.4 \%$. There was no husband or caregivers who refused to be interviewed.

\subsection{Characteristics of Pregnant Women}

The average age of pregnant women was $26.0 \pm 5.6$ years. Among pregnant women 118 (39.2\%) had less than two deliveries and 137 (45.5\%) experienced at least two prior pregnancies.

Almost all pregnant women (293, 97.3\%) were married. Farmers and housewives represented respectively 30.2\% $(n=91)$ and $22.6 \%(n=68)$. Low education levels were common, with no schooling $(85,28.2 \%)$ or primary education only $(136,42.5 \%)$ as the highest education level for a considerable proportion of women.

A proportion of $58.1 \%(\mathrm{n}=175)$ of pregnant women were Christians and $112(37.2 \%)$ practiced traditional religion. In $71.4 \%(\mathrm{n}=125)$ of women, the power of decision belonged to men.

Among pregnant women, 159 (52.8\%) showed a low level of knowledge on ANC visits and $122(40.5 \%)$ a poor knowledge about danger signs of pregnancy, 109 (36.2\%) declared the period of the first ANC visit is between the $4^{\text {th }}$ and $5^{\text {th }}$ month of pregnancy and $159(52.8 \%)$ of them did not have family support for the first ANC visit.

\subsection{Utilization of Antenatal Care Services during the First Trimester of Pregnancy}

Among the 301 pregnant women, 24.6\% attended ANC services in the first trimester of pregnancy.

\subsection{Determinants of the Low Utilization of CPN during the First Trimester of Pregnancy}

\subsubsection{Univariate Analysis}

Tables $1 \mathrm{a}$ and $\mathrm{Ib}$ summarize determinants of the low utilization of ACN during the first trimester of pregnancy. As shown in table 1a, age, education, occupation, knowledge relatives to ANC, number of prior pregnancies an deliveries influenced utilization of ANC, As per table 1b, absence of family support, lack of exposure to media, patient-welcome appreciation, waiting time, transportation modes, participation to behavior change communication for antenatal care and the qualification of the health personnel affected utilization of ANC.

Marital status, ethnicity, religion, decision-making power, knowledge of danger signs of pregnancy, distance between home and health center, costs of ANC, existence of barriers to reach ANC services, availability of ANC providers, respect of women's intimacy and interpersonal communication were not associated with low utilization of ACN during the first trimester of pregnancy.

\subsubsection{Multivariate Analysis}

Among factors significantly associated with lower use of ANC services in the univariate analysis, five (education levels, knowledge of the required period of the first ANC visit during pregnancy, knowledge of the benefits of ANC, participation in meetings of communication for behavior change and patient-welcome appreciation) remained significantly associated with the dependent variable in multivariate analysis.

Level of education: Women with no schooling or primary school level showed a higher likelihood for low utilization of ANC services during the first trimester of pregnancy compared to those with more education (secondary and above) controlling for other factors: $\mathrm{OR}=3.293[1.534$; 7.071].

Knowledge of the required period of the first ANC visit: 
women with a wrong knowledge of the required period of the first ANC visit had a higher likelihood (five times) to underutilize ANC services than their counterparts controlling for other factors: $\mathrm{OR}=5.131[1.972-13.353]$.

Knowledge of the benefits of ANC: Pregnant women with low knowledge of the benefits of ANC showed a higher likelihood (about four times) of underutilization of ANC services compared to those with low to medium knowledge controlling for other factors: $\mathrm{OR}=4.031$ [1.401 - 11.594].

Participation in communication for behavioral change sessions: Communication sessions for behavioral change are common offering in ANC in Benin and women who participated in communication for behavioral change sessions were less likely to experience low utilization of ANC services than their counterparts: $\mathrm{OR}=0.059[0.028-$ $0.125]$.

Patient-welcome appreciation: pregnant women who felt that patient-welcome was adequate had a low likelihood to experience the low utilize ANC during the first trimester of pregnancy, compared with those who felt the opposite adjusting on all other factors: $\mathrm{OR}=0.162[0.051-0.513]$

Table 1a. Determinants of the low utilization of the ANC during the first trimester of pregnancy in Athiémé municipality, (univariate analysis), southern Benin 2014.

\begin{tabular}{|c|c|c|c|c|c|}
\hline \multirow{2}{*}{ Independent variables } & \multicolumn{5}{|c|}{ Utilization of ANC during the first trimester of pregnancy } \\
\hline & Low & adequate & OR & $95 \% \mathrm{CI}$ & P-value \\
\hline Age (years) & & & & & 0.042 \\
\hline$\geq 20$ & 202 & 59 & 2.05 & {$[1.02-4.15]$} & \\
\hline$<20$ & 25 & 15 & 1 & & \\
\hline Occupation & & & & & $<0.001$ \\
\hline Others occupations & 216 & 60 & 4.58 & {$[1.98-10.6]$} & \\
\hline Pupils/students/apprentices & 11 & 14 & 1 & & \\
\hline Education & & & & & $<10^{-5}$ \\
\hline No schooling and primary school & 185 & 40 & 3.74 & {$[2.04-6.87]$} & \\
\hline High school or university & 42 & 34 & 1 & & \\
\hline Number of deliveries & & & & & $<0.001$ \\
\hline$\geq 1$ & 190 & 46 & 3.13 & {$[1.67-5.86]$} & \\
\hline 0 & 37 & 28 & 1 & & \\
\hline Number or pregnancies & & & & & $<0.001$ \\
\hline$\geq 2$ & 193 & 48 & 3.07 & {$[1.62-5.85]$} & \\
\hline 1 & 34 & 26 & 1 & & \\
\hline Knowledge of the benefits of $\mathrm{ACN}$ & & & & & 0.0047 \\
\hline Low and medium & 204 & 57 & 2.65 & {$[1.25-5.57]$} & \\
\hline High & 23 & 17 & 1 & & \\
\hline Knowledge of diseases to prevent during pregnancy & & & & & $<0.001$ \\
\hline Low & 139 & 27 & 2.75 & {$[1.41-4.91]$} & \\
\hline Medium or high & 88 & 47 & 1 & & \\
\hline $\begin{array}{l}\text { Knowledge of required period of the first ANC visit } \\
\text { for pregnant women }\end{array}$ & & & & & $<10^{-8}$ \\
\hline$>3$ months & 109 & 10 & 5.91 & [2.77 - 12.94] & \\
\hline$\leq 3$ months & 118 & 64 & 1 & & \\
\hline
\end{tabular}


Table 1b. Determinants of the low utilization of the ANC during the first trimester of pregnancy in Athiémé municipality, (univariate analysis), southern Benin 2014

\begin{tabular}{|c|c|c|c|c|c|}
\hline \multirow{2}{*}{ Independent variables } & \multicolumn{5}{|c|}{ Utilization of ANC during the first trimester of pregnancy } \\
\hline & Low & adequate & OR & $95 \% \mathrm{CI}$ & P-value \\
\hline Family support & & & & & $<0.001$ \\
\hline No & 134 & 25 & 2.82 & {$[1.58-5.08]$} & \\
\hline Yes & 93 & 49 & 1 & & \\
\hline Exposure to media & & & & & 0.0052 \\
\hline No & 56 & 7 & 3.13 & {$[1.33-8.54]$} & \\
\hline Yes & 171 & 67 & 1 & & \\
\hline Exposure to TV & & & & & 0.001 \\
\hline No & 162 & 37 & 2.49 & {$[1.40-4.43]$} & \\
\hline Yes & 65 & 37 & 1 & & \\
\hline $\begin{array}{l}\text { Organization of behavior change communication for } \\
\text { antenatal care }\end{array}$ & & & & & $<0.001$ \\
\hline No & 128 & 11 & 7.40 & {$[3.55-15.76]$} & \\
\hline Yes & 99 & 63 & 1 & & \\
\hline $\begin{array}{l}\text { Participation to behavior change communication for } \\
\text { antenatal care }\end{array}$ & & & & & $<0.001$ \\
\hline No & 195 & 19 & 17.64 & {$[8.88-35.41]$} & \\
\hline Yes & 32 & 55 & 1 & & \\
\hline Transportation modes & & & & & 0.011 \\
\hline Walking & 145 & 35 & 1.97 & {$[1.12-3.47]$} & \\
\hline Car / Motorbike / Bike & 82 & 39 & 1 & 1 & \\
\hline Waiting time & & & & & 0.009 \\
\hline$\geq 4 h$ & 76 & 13 & 2.36 & {$[1.17-4.83]$} & \\
\hline$<4 \mathrm{~h}$ & 151 & 61 & 1 & & \\
\hline Patient-welcome appreciation & & & & & 0.006 \\
\hline Poor & 55 & 7 & 3.06 & {$[1.33-7.06]$} & \\
\hline Adequate & 172 & 67 & 1 & & \\
\hline Qualification of care provider & & & & & 0.027 \\
\hline Nurse-assistant or nurse & 163 & 43 & 1.84 & {$[1.03-3.28]$} & \\
\hline Midwife & 64 & 31 & 1 & & \\
\hline
\end{tabular}

Table 2. Determinants of the low utilization of ANC services during the first trimester of pregnancy in Athiémé municipality (mulita variate final model), southern Benin 2014

\begin{tabular}{cccc}
\hline Variables & p-value & OR & 95\%CI \\
\hline $\begin{array}{c}\text { Education level (no schooling and primary school } \\
\text { /high school or university) }\end{array}$ & 0.002 & 3.293 & {$[1.534-7.071]$} \\
$\begin{array}{c}\text { Knowledge of required period of the first ANC } \\
\text { visit for pregnant women (>3 months/ } \mathbf{3} \text { months) }\end{array}$ & 0.001 & 5.131 & {$[1.972-13.353]$} \\
$\begin{array}{c}\text { Knowledge of the benefits of ACN (low an } \\
\text { medium/high) }\end{array}$ & 0.010 & 4.031 & {$[1.401-11.594]$} \\
$\begin{array}{c}\text { Participation to behavior change communication } \\
\text { for antenatal care (Yes/No) }\end{array}$ & $<0.001$ & 0.059 & {$[0.028-0.125]$} \\
Patient-welcome appreciation (Adequate/Poor) & 0.002 & 0.162 & $0.513]$ \\
\hline
\end{tabular}




\section{Discussion}

The present study explored the determinants of low utilization of ANC services during the first trimester of pregnancy. Data were collected using a combination of techniques and tools, qualitative and quantitative analysis were performed. This strengthened the quality of our results. Administration of the questionnaire to pregnant women was conducted by trained investigators to minimize bias of information. Data collection tools were pre-tested. Regular supervision helped to ensure that the collection procedures were properly followed. Debriefing sessions were organized every noon. All these ensured the validity of data collected.

\subsection{Use of ANC Services during the First Trimester of Pregnancy}

The rate of utilization of ANC services during the first trimester of pregnancy in this study was low. Several studies support this result. Indeed, Onoh and Dennis et al. in Nigeria, reported $16.9 \%$ and $28.4 \%$ respectively $[11,12]$. In one study conducted by Nicholas in Zambia, $17 \%$ of pregnant women had used ANC services in the first trimester of pregnancy [13]. In contrast, Osungbade in Nigeria reported a very low rate of utilization of ANC services (7.2\%) in the first trimester of pregnancy [14]. According to Nyei Zambia, $19 \%$ of women achieved ANC visit in the first trimester of pregnancy [15]. Sudan Ali observed a higher rate of 59.6\% [1]. The plausible explanation for the lack of utilization of ANC in the first trimester are mothers waiting for the pregnancy to show, myths surrounding revelation of early pregnancy and other factors relative to health system as discussed below.

\subsection{Factors Related to the Low Utilization of ANC Services during the First Trimester of Pregnancy}

In the present study, women with low education levels were less likely to use ANC services during the first trimester compared to women with more education (secondary and above) controlling for other factors. This result was in convergence with studies conducted by other authors $[12 ; 16$; 17]. Indeed, women less educated, have less knowledge about ANC and will more unlike to use ANC.

Age was associated with low utilization of ANC services during the first trimester of pregnancy, although it was not remained significant in the final model. Thus, women aged 20 years and over showed little interest in the use of ANC services and therefore did not fulfill ANC visits compared to younger women. This result is consistent with those reported by Regassa in Ethiopia [16]. Our results were different from those observed by Feijen de Jong et al. [17]. This could be due to fear, lack of experience of pregnant younger women.

Number of deliveries and pregnancies were statistically associated with the low utilization of ANC services during the first trimester of pregnancy, although they were not independent determinants. In the present study, women who have already given birth at least once, showed a little interest in the timely utilization of ANC services compared to those who never gave birth. Also, women who had been pregnant more than once, compared with those who were first-time pregnant, showed little interest to timely ANC visits. Indeed it was 3.1 times more likely to find at least one woman who experienced prior pregnancies and underutilize ANC services than women with first pregnancy. These results are consistent with several studies $[18 ; 19 ; 20 ; 21]$. This could be explained by the fact that women who experienced prior pregnancies or deliveries had capitalized these experiences. Therefore, they are less likely to comply with timely ANC visits.

In the present study, women who did not know the required period of the first ANC used about 5.91 times less ANC services than those who showed adequate knowledge. In fact, $39.53 \%$ of pregnant women did not know the required period of the first ANC visit. This is consistent with studies conducted in Tanzania, Nigeria and Uganda $[1 ; 11$; 22].

There was a link between knowledge of the benefits of ANC and their utilization. Thus, the low utilization of ANC services during first trimester of pregnancy was significantly related to poor knowledge of the benefits of ANC.

The participation of women in communication sessions for behavioral change was statistically linked to the low utilization of ANC services during the first trimester of pregnancy. Thus, women who have not attended these sessions showed about 17.64 times higher risk of low utilization of ANC compared with those who attended these meetings.

There was a significant relationship between family support and low utilization of ANC services during the first trimester of pregnancy. But this factor was not an independent determinant. Indeed, women with lack of family support to attend communications' sessions for behavioral changes utilized ANC services less than those who attended these sessions. This result was in convergence with the study conducted by Gross in Tanzania [1].

Most of pregnant women thought that ANC visits are an opportunity to be informed about both their health and the evolution of pregnancy. This adequate perception was similar to those reported by Mrisho et al. in Tanzania [23]. But some women believed that if the pregnant woman feels no problem with her pregnancy, there is no reason to attend earlier health centers. Some studies in Uganda and Nigeria reported similar results $[1 ; 21 ; 23]$.

The appreciation of the cost of ANC, the distance between home and the health center and the existence of some barriers to access Health centers were not statistically associated with the low utilization of ANC services. Transportation modes were associated with low utilization of ANC services. Pregnant women who were on foot, showed low utilization of ANC services compared to those who came to the health center by car or motorcycle. This could be explained by the fact that the greater the ease of access to the center, the better women will utilize ANC services [24; 25]. 
Patient-welcome appreciation was an independent determinant the low utilization of ANC services during the first trimester of pregnancy. Thus, women who felt that patient-welcome was poor underutilize ANC services compared to those who felt it adequate. This result was in accordance with the Gross and al. report [1]. Pregnant women referred to the poor patient-welcome as a barrier to the utilization of ANC services. Indeed, a qualitative study in women and men regarding experiences of disrespectful maternity care and abuse during childbirth in Morogoro Region, Tanzania reported several types of disrespect such as feeling ignored or neglected, monetary demands or discriminatory treatment, verbal and physical abuse [26]. According to $\mathrm{WHO}$, respectful maternity care are needed to stop physical abuse, non-consented care, non-confidential care, non-dignified care (including verbal abuse), discrimination based on specific attributes, abandonment or denial of care and detention in facilities experienced by women during ANC and childbirth [4].

Cultural beliefs influenced influenced pregnant women's habits to the extent that they felt that when pregnancy was too young, it is advised to hide it to the mystical forces do not destroy it. This result was similar to several reports [11; $22 ; 23]$. This result was also consistent with findings from the interviews with the matrons, focus group with women's husbands, interviews with health qualified care providers.

Other women had claimed financial constraints. The decision to attend ANC visits was jointly take by both spouses as suggested by Denis and colleagues in study conducted in Nigeria [12].

\section{Conclusions}

ANC services were underutilized in Athiémé, a southern Benin rural setting. Determinants of the low utilization of ANC services identified were low education level, ignorance of required period of the first ANC visit for pregnant women, and ignorance of the benefits of ANC and poor patient-welcome quality. Participation to behavior changes communication for antenatal care promoted ANC utilization in. Overall, Improvement of ANC utilization is needed. Communication strategies to strengthen knowledge on ANC, behavior change and respectful maternity care need to be promoted as well as improvement of access to ANC services is critical in order to achieve optimal maternity outcomes.

\section{Conflicts of Interest}

The authors declare no conflict of interest.

\section{Acknowledgements}

The authors thank the authorities of Athiémé municipality and participants in the study.

\section{REFERENCES}

[1] Gross K, Alba S, Glass TR, Schellenberg JA, Obrist B. Timing of antenatal care for adolescent and adult pregnant women in south-eastern Tanzania. BMC Pregnancy Childbirth. Vol 12, No16, 1-12; doi: 10.1186/1471-2393-12-16. 2012.

[2] National Institute of statistics and economic analysis (Benin). Demographic and health and multiple indicators' survey EDSN-MICS-IV 2012: Final report. Cotonou: INSAE, 2012.

[3] Trinh LT, Rubin G. Late entry to antenatal care in New South Wales, Australia. Reprod Health., Vol.3, No8,1-8; doi:10.1186/1742-4755-3-8 2006

[4] World Health Organization, Respectful maternity care, the universal rights of childbearing women. Available on http://www.who.int/woman_child_accountability/ierg/report s/2012 01S Respectful Maternity Care Charter The Univ ersal_Rights_of_Childbearing_Women.pdf (Accessed on 29 August 2015).

[5] Maghrebi H, Ben Mouhoub N, Miguil M. Maternal Mortality in North Africa: Contribution of Anaesthetists of Maghreb, 2010 .

[6] Fatembo HV. Determinants of low effective refocused antenatal care coverage in the municipality of Bohicon Benin. Public Health Master memory. IRSP; Ouidah; 2012

[7] National Institute of statistics and economic analysis Institut National de Statistique et de l'Analyse Economique (Bénin). National census' results, RGPH 4; 2013.

[8] Thadeus S, Maine D, Too far to walk: maternal mortality in context. Social Science and Medicine, Vol. 38, N8, 10911110,1994

[9] Ministry of Decentralization and Local Government, Monograph of commune of Athiémé. Mission of decentralization of Communes, Cotonou, 2006.

[10] Hsieh, F.Y, Block, D.A, Larsen, M.D. A Simple Method of Sample Size Calculation for Linear and Logistic Regression. Statistics in Medicine, Vol. 17, Nº14, 1623-1634; 1998

[11] Onoh RC, Umeora OUJ, Agwu UM, Ezegwui HU, Ezeonu PO, Onyebuchi AK. Pattern and Determinants of Antenatal Booking at Abakaliki Southeast Nigeria. Ann Med Health Sci Res. Vol. 2, N², 169-75, 2012.

[12] Dennis II, Terkimbi UB. Gestational age at booking for antenatal care in a tertiary health facility in north-central, Nigeria. Niger Med J., Vol.53, Nº4, 236-43, 2012

[13] Nicholas N, Kyei A, Oona M, Campbell R, Gabrysch S. The Influence of Distance and Level of Service Provision on Antenatal Care Use in Rural Zambia. PLoS One. Vol.7, $\mathrm{N}^{\circ} 10$, 1-10, e46475, 2012,

[14] Osungbade K, Oginni S, Olumide A. Content of antenatal care services in secondary health care facilities in Nigeria: implication for quality of maternal health care. Int $\mathrm{J}$ Qual Health Care, Vol.20, N5, 346-51, 2008.

[15] Kyei NN, Chansa C, Gabrysch S. Quality of antenatal care in Zambia: a national assessment. BMC Pregnancy Childbirth.Vol.3, N 12 ,doi: 10.1186/1471-2393-12-15, 2012

[16] Ali AA1, Osman MM, Abbaker AO, Adam I. Use of antenatal 
care services in Kassala, eastern Sudan. BMC Pregnancy Childbirth, Vol.10, №67, 1-4; doi: 10.1186/1471-2393-10-67, 2010.

[17] Yesuf EA, Calderon-Margalit R. Disparities in the use of antenatal care service in Ethiopia over a period of fifteen years. BMC Pregnancy Childbirth, Vol. 13, $\mathrm{N}^{\circ} 1,1-10$ doi: 10.1186/1471-2393-13-131, 2013

[18] Tran TK, Gottvall K, Nguyen HD, Ascher H, Petzold M. Factors associated with antenatal care adequacy in rural and urban contexts-results from two health and demographic surveillance sites in Vietnam. BMC Health Serv Res. Vol.12, Nº40. doi: 10.1186/1472-6963-12-40, 2012

[19] Regassa N. Antenatal and postnatal care service utilization in southern Ethiopia: a population-based study. Afr Health Sci., Vol.11, N³, 390-7. 2011

[20] Feijen-de Jong EI, Jansen DE, Baarveld F, van der Schans CP, Schellevis FG, Reijneveld SA. Determinants of late and/or inadequate use of prenatal healthcare in high-income countries: a systematic review. Eur J Public Health. Vol.22, $\mathrm{N}^{\circ} 6,904-13,2012$

[21] Andrew EV, Pell C, Angwin A, et al. Factors affecting attendance at and timing of formal antenatal care: results from a qualitative study in madang, papua new Guinea. PLoS One. Vol.9, $\mathrm{N}^{\circ}$ 5, e93025, 2014.
[22] Simkhada B, Porter MA, van Teijlingen ER. The role of mothers-in-law in antenatal care decision-making in Nepal: a qualitative study. BMC Pregnancy Childbirth. Vol.10, N³4, doi: 10.1186/1471-2393-10-34, 2010.

[23] Mrisho M, Obrist B, Schellenberg JA, Haws RA, Mushi AK, Mshinda H, Tanner M, Schellenberg D. The use of antenatal and postnatal care: perspectives and experiences of women and health care providers in rural southern Tanzania. BMC Pregnancy Childbirth. Vol.9, N¹0. doi: 10.1186/1471-2393-9-10

[24] Ebeigbe PN, Igberase GO. Reasons given by pregnant women for late initiation of antenatal care in the Niger delta, Nigeria. Ghana Med J. Vol.44, N², 47-51, 2010.

[25] Tsegay Y, Gebrehiwot T, Goicolea I, Edin K, Lemma H, Sebastian MS. Determinants of antenatal and delivery care utilization in Tigray region, Ethiopia: a cross-sectional study. Int J Equity Health, Vol.12, No30. doi: 10.1186/1475-9276-12-30, 2013

[26] McMahon S.A, George A.S., Chebet J.J., Mosha I.H., Mpembeni R. NM., Winch P.J.. Experiences of and responses to disrespectful maternity care and abuse during childbirth; a qualitative study with women and men inMorogoro Region, Tanzania. BMC Pregnancy and Childbirth, Vol.14, N²68, 2014, doi:10.1186/1471-2393-14-268 